\title{
Choline, an essential nutrient for humans
}

\author{
STEVEN H. ZEISEL ${ }^{*}, 1$, KERRY-ANN DA COSTA, ${ }^{*}$ PETER D. FRANKLIN, ${ }^{\dagger}$ EDWARD A. ALEXANDER, ${ }^{\ddagger}$ \\ J. THOMAS LAMONT,' NANCY F. SHEARD," AND ALEXA BEISER" \\ 'Department of Nutrition, University of North Carolina at Chapel Hill, Chapel Hill, North Carolina 27599-7400, USA; ${ }^{\dagger}$ Department \\ of Radiology, University Hospital, Boston University School of Medicine, Boston, Massachusetts 02118, USA; 'Department of Medicine, \\ Renal Division, Boston City Hospital, Boston University School of Medicine, Boston, Massachusetts 01003, USA; 'Department of \\ Medicine, Section of Gastroenterology, University Hospital, Boston University School of Medicine, Boston, Massachusetts 02118, USA; \\ "Department of Food Science and Nutrition, University of Massachusetts at Amherst, Amherst, Massachusetts 01003, USA; and \\ 'Department of Epidemiology and Biostatistics, Baston University School of Public Health, Boston, Massachusetts 02118, USA
}

\begin{abstract}
Choline is required to make essential membrane phospholipids. It is a precursor for the biosynthesis of the neurotransmitter acetylcholine and also is an important source of labile methyl groups. Mammals fed a choline-deficient diet develop liver dysfunction; however, choline is not considered an essential nutrient in humans. Healthy male volunteers were hospitalised and fed a semisynthetic diet devoid of choline supplemented with $500 \mathrm{mg} /$ day choline for 1 wk. Subjects were randomly divided into two groups, one that continued to receive choline (control), and the other that received no choline (deficient) for three additional wk. During the 5 th wk of the study all subjects received choline. The semisynthetic diet contained adequate, but no excess, methionine. In the choline-deficient group, plasma choline and phosphatidylcholine concentrations decreased an average of $30 \%$ during the 3 -wk period when a choline-deficient diet was ingested; plasma and erthrocyte phosphatidylcholine decreased 15\%; no such changes occurred in the control group. In the cholinedeficient group, serum alanine aminotransferase activity increased steadily from a mean of $0.42 \mu \mathrm{kat} /$ liter to a mean of $0.62 \mu$ kat/liter during the 3-wk period when a choline-deficient diet was ingested; no such change occurred in the control group. Other tests of liver and renal function were unchanged in both groups during the study. Serum cholesterol decreased an average of $15 \%$ in the deficient group and did not change in the control group. Healthy humans consuming a choline-deficient diet for 3 wk had depleted stores of choline in tissues and developed signs of incipient liver dysfunction. Our observations support the conclusion and choline is an essential nutrient for humans when excess methionine and folate are not available in the diet. Z Zeisel, S. H.; da Costa, K.-A.; Franklin, P. D.; Alexander, E. A.; LaMont, J. T.; Sheard, N. F.; Beiser, A. Choline, an essential nutrient for humans. FASEB J. 5: 2093-2098; 1991.
\end{abstract}

Key Words: choline deficiency - lipotropes - human - liver - kidney - nutrient requirements

Choline IS REQUiRed to MAKe the phospholipids phosphatidylcholine, lysophosphatidylcholine, choline plasmalogen, and sphingomyelin - essential components of all membranes. It is a precursor for the biosynthesis of the neurotransmitter acetylcholine and also is an important source of labile methyl groups (1). Much attention has been given to the effects of supplemental choline upon brain function where it has been suggested that such treatments enhance acetylcholine synthesis and release (2-4). Whether choline is normally required as part of the human diet is still unresolved.
Several lines of evidence suggest that choline might be an essential nutrient for humans. In many other mammals, including the monkey and rat, choline deficiency results in liver and renal dysfunction (1). Human cells grown in culture have an absolute requirement for choline (5). Malnourished humans have diminished plasma or serum choline concentrations even when fed parenterally (6-8), and humans fed by vein with solutions containing little or no choline develop liver dysfunction similar to that seen in choline-deficient animals $(6,9,10)$. Chawla et. al. (7) have suggested that choline is an essential nutrient for malnourished humans.

Choline is considered a dispensable nutrient for humans because: 1) There is an endogenous pathway for the de novo biosynthesis of choline moiety via the sequential methylation of phosphatidylethanolamine (11), 2) It has been difficult to identify a choline deficiency syndrome in healthy humans as most common foods contain choline and the demand for choline is modified by the rate of growth of an individual and by complex interrelationships between choline and the nutrients methionine, folic acid, and vitamin $\mathrm{B}_{12}$ (lipotropes) (1), and 3) No one has tried to experimentally induce choline deficiency in normal humans.

These arguments do not prove or disprove that humans require dietary choline. Diminished tissue levels of a nutrient associated with removal of the nutrient from the diet suggest a nutrient requirement, but deficiency should be associated with deterioration of organ function if a nutrient is essential. The presence of a pathway for endogenous synthesis does not make a nutrient dispensable. Most mammals can synthesize choline moiety, yet they become severely (often fatally) ill if deprived of choline when excess methionine is not available (1). In this study we fed choline-deficient diets to normal humans, and report changes in the choline and phosphatidylcholine content of blood and in liver function.

\section{METHODS}

\section{Subjects}

Sixteen healthy male volunteers were recruited for this study with their informed consent. The study was approved by the Institutional Review Board of the Boston University Research Center. Subjects were excluded if they consumed more than $1 \mathrm{oz}$. alcohol per day or if they had any preexisting disease. Subjects were randomized into a control group $(n=7)$ or a choline-deficient group $(n=8)$. One control

'To whom correspondence should be addressed, at: Department of Nutrition, University of North Carolina at Chapel Hill, CB \#7400 McGavran-Greenberg Hall, Chapel Hill, NC 27599-7400, USA. 
subject was recruited and began the study, but was dropped because on day 1 liver function tests were abnormal. Seven other subjects were recruited but never began the study because of abnormal clinical chemistry values on initial screening or because they changed their decision to participate. The mean age of the remaining six control subjects was 26.8 years $( \pm 1.5$ SEM) and the mean age of the eight cholinedeficient subjects was 29.1 years $( \pm 1.8)$. The mean weight for the control group was $74.4 \mathrm{~kg}( \pm 4.9)$, whereas the cholinedeficient group mean weight was $72.4 \mathrm{~kg}( \pm 4.5)$. None of the subjects was on medication, and none had a history of chronic illness.

\section{Study design}

Subjects were admitted to the Boston University Clinical Research Center and were observed constantly during the 5 wk of the study (two control subjects and one deficient subject were not constantly observed, but consumed all meals in the presence of our dietician). During the first wk of the study all subjects consumed the same choline-containing diet. During the middle 3 wk of the study the control group continued on the choline-containing diet while the choline deficient group consumed the same diet without choline. During the 5th wk of the study all subjects consumed the choline-containing diet. The subjects consumed three isocaloric meals each day consisting of a liquid diet delivering daily protein $\left(0.95 \mathrm{~g} \cdot \mathrm{kg}^{-1} \cdot \mathrm{day}^{-1}\right.$; delivered at least the recommended daily allowance $[\mathrm{RDA}]^{2}$ for all essential amino acids) as well as a portion of daily energy intake. An evening snack consisting of protein-free cookies and ginger ale was also provided. Subjects ingested $40 \mathrm{kcal} \cdot \mathrm{kg}^{-1} \cdot$ day $^{-1}(9.5 \%$ protein, $35 \%$ fat, $55.5 \%$ carbohydrate). The dietary protein source was a purified soybean $\alpha$-soy protein (STA-PRO 3200, Central Soya Company; Fort Wayne, Ind.). Fat was supplied as safflower oil, carbohydrate as dextrose, and fiber as cellulose (Avicel; FMC Corporation, Philadelphia, Pa.). The diet delivered the recommended daily allowance for all amino acids, vitamins, and minerals and contained (per 70 $\mathrm{kg}$ body weight/day) $13 \mathrm{mg}$ of total choline, $9 \mu \mathrm{g}$ of vitamin $\mathrm{B}_{12}, 305 \mu \mathrm{g}$ of folate, $921 \mathrm{mg}$ of L-methionine, and $834 \mathrm{mg}$ of L-cystine (amino acid composition as determined by manufacturer - all subjects' diets were prepared using a single lot of protein; vitamin content calculated based on addition of vitamins to diet; choline content per determination in our laboratory). The control (choline-supplemented) subjects received $700 \mathrm{mg} /$ day choline chloride as capsules. The choline-deficient subjects received placebos (cellulose) during the experimental period (middle $3 \mathrm{wk}$ ) but received the choline capsules during the first and last weeks of the study.

Blood was obtained by venipuncture before the start of the study, and thereafter on days $7,10,14,21,28$, and 35 when subjects were fasting in the morning. Blood samples for choline and phosphatidylcholine analyses were placed on ice immediately after being drawn, and were centrifuged at $3000 \times \mathrm{g}$ for $15 \mathrm{~min}$ at $4^{\circ} \mathrm{C}$ within $30 \mathrm{~min}$ of being collected. Blood drawn for other measurements was sent to a clinical laboratory for analysis (Smith Kline Bio-Science Laboratories, Waltham, Mass.). The first morning voiding of urine was collected on each of the above days and subjected to urinalysis and microscopic examination. A sample of this urine was sent to the clinical laboratory (Smith Kline Bio-Science Laboratories) for creatinine determination.

\section{Analytical methods}

Choline was determined using a gas chromatograph/mass spectrometric method that we have previously published (12,
13). Phosphatidylcholine was determined by extracting plasma or erythrocytes using the method of Bligh and Dyer (14), isolating phosphatidylcholine by thin-layer chromatography (12), and measuring phosphorus content of the phosphatidylcholine band (15).

Serum alanine aminotransferase (ALT), aspartate aminotransferase (AST), rglutamyl transpeptidase (GGT), lactic dehydrogenase (LDH), alkaline phosphatase, urea nitrogen (BUN), total and direct bilirubin, albumin, total protein, triglyceride, total cholesterol, HDL cholesterol, and urine creatinine were measured using an automated procedure (Technicon SMAC) by the clinical laboratory (Smith Kline Bio-Science). Prothrombin time and partial thromboplastin time were measured using photo-optical clot determination (MLA 700) by the clinical laboratory (Smith Kline BioScience). Urinalyses and microscopic examinations of urine were performed by Dr. Alexander.

Computerized tomography of the liver was performed on days 7 and 28 of the study. Density measurements were performed using a region of interest circle and the mean density of the area was recorded for the right anterior, right posterior, left medial, and left lateral lobes. Values for all lobes were averaged and data were expressed in Houndsfield units. Liver size was estimated by measuring the lateral dimension at the level of the portal vein from the liver edge to the midline (dimen sion A), and anterior-posterior dimension by measuring from the posterior to the anterior liver edge at the level of the portal vein (dimension $B$ ). Data are described as the product $A \times B\left(\mathrm{~cm}^{2}\right)$.

\section{Statistics}

The primary outcome measures were plasma choline and phosphatidylcholine and serum ALT activity, expressed as a change from day 7 to day 28. Additional variables were measured and analyzed for descriptive purposes. Twosample $t$-tests were used to compare baseline mean values of the outcome variables on day 7 and to compare the mean change in these variables between days 7 and 28. Analyses were performed using statistical analysis system (SAS) software on an IBM personal computer.

\section{RESULTS}

Subjects consuming either diet had normal physical examinations throughout the study, all maintained their body weights, and no subject complained of illness. Both groups had similar $(P<0.05)$ normal values for all of the clinical chemistry measurements on day 0 and day 7 (after 1 wk of consuming the standardized choline-containing diet; Table 1).

Plasma choline concentrations remained relatively constant in the control group (9.6-10.9 $\mu \mathrm{mol} /$ liter) during the 5-wk study (Fig. 1). In the choline-deficient group, mean plasma choline concentration decreased approximately $30 \%$ during the 3-wk period when a choline-deficient diet was ingested (Fig. 1; $P<0.01$ change in choline-deficient group compared with change in control group was significant by

\footnotetext{
${ }^{2}$ Abbreviations: RDA, recommended daily allowance; ALT, alanine aminotransferase; AST, aspartate aminotransferase; GGT, glutamyl transpeptidase; LDAH, lactic dehydrogenase; BUN, urea nitrogen; SAS, statistical analysis system; PeMT, phosphatidylethanolamine- $\mathrm{N}$-methyltransferase; VLDL, very low density lipoprotein; TPN, total parenteral nutrition.
} 
TABLE 1. Change in value of clinical chemistries between day 7 and day 28 (period of experimental diet ingestion) ${ }^{a}$

\begin{tabular}{|c|c|c|c|c|}
\hline & \multicolumn{2}{|c|}{ Controls } & \multicolumn{2}{|c|}{ Deficients } \\
\hline & Day 7 value & Day 28-day 7 & Day 7 value & Day 28-day 7 \\
\hline Serum ALT, $\mu$ kat/liter & $0.40( \pm 0.1)$ & $-0.08( \pm 0.12)$ & $0.42( \pm .08)$ & $+0.22( \pm 0.07)^{b}$ \\
\hline Serum AST, $\mu \mathrm{kat} / \mathrm{liter}$ & $0.28( \pm 0.03)$ & $0( \pm 0.05)$ & $0.32( \pm 0.05)$ & $+0.10( \pm 0.07)$ \\
\hline Serum GGT, $\mu \mathrm{kat} / \mathrm{liter}$ & $0.40( \pm 0.17)$ & $-0.03( \pm 0.05)$ & $0.48( \pm 0.13)$ & $-0.10( \pm 0.03)$ \\
\hline Serum bile acids, $\mu \mathrm{mol} / \mathrm{liter}$ & $1.0( \pm 0.3)$ & $+0.2( \pm 0.3)$ & $1.3( \pm 0.5)$ & $+0.4( \pm 0.5)$ \\
\hline Serum total bilirubin, $\mu \mathrm{mol} / \mathrm{liter}$ & $13.7( \pm 1.7)$ & $-1.7( \pm 1.7)$ & $13.7( \pm 1.7)$ & $0( \pm 1.7)$ \\
\hline Serum direct bilirubin, $\mu \mathrm{mol} / \mathrm{liter}$ & $2.6( \pm 0.7)$ & $-1.7( \pm 0.3)$ & $1.7( \pm 0.7)$ & $0( \pm 0.3)$ \\
\hline Serum albumin, $\mathrm{g} / \mathrm{liter}$ & $46( \pm 1)$ & $-2( \pm 1)$ & $47( \pm 1)$ & $-2( \pm 2)$ \\
\hline Serum total protein, $\mathrm{g} / \mathrm{liter}$ & $69( \pm 2)$ & $-3( \pm 2)$ & $71( \pm 1)$ & $-3( \pm 2)$ \\
\hline Prothrombin time, $\mathrm{s}$ & $11.6( \pm 0.2)$ & $0( \pm 0.3)$ & $11.7( \pm 0.2)$ & $+0.1( \pm 0.2)$ \\
\hline Serum creatinine, $\mu \mathrm{mol} / \mathrm{liter}$ & $97( \pm 4)$ & $0( \pm 9)$ & $97( \pm 5)$ & $0( \pm 4)$ \\
\hline Urine creatinine, $\mu \mathrm{mol} / \mathrm{liter}$ & $115( \pm 18)$ & $0( \pm 9)$ & $133( \pm 36)$ & $-35( \pm 35)$ \\
\hline Serum total cholesterol, $\mathrm{mmol} / \mathrm{liter}$ & $4.34( \pm 0.41)$ & $-0.10( \pm 0,05)$ & $4.68( \pm 0.36)$ & $-0.72( \pm 0.16)^{6}$ \\
\hline Serum HDL cholesterol, $\mathrm{mmol} /$ liter & $0.93( \pm 0.03)$ & $-0.08( \pm 0.05)$ & $1.06( \pm 0.08)$ & $-0.13( \pm 0.08)$ \\
\hline Serum LDL cholesterol, mg/dl & $2.84( \pm 0.31)$ & $-0.03( \pm 0.05)$ & $2.92( \pm 0.31)$ & $-0.47( \pm 0.18)$ \\
\hline Serum trigylceride, $\mathrm{mg} / \mathrm{dl}$ & $1.29( \pm 0.29)$ & $+0.02( \pm 0.22)$ & $1.55( \pm 0.27)$ & $-0.28( \pm 0.16)$ \\
\hline
\end{tabular}

${ }^{a}$ Data are expressed as mean \pm SEM. ${ }^{b} P<0.05$ significant difference day 28-day 7 value by two-sample $t$ test. difference day 28-day 7 value by two-sample $t$ test.

two-sample $t$ test). When the choline-deficient group was switched to a choline-sufficient diet during the last week of the study, plasma choline concentrations returned to baseline (Fig. 1; $P<0.001$ that day 35 value would be different from day 28 value by $t$ test; day 35 value in deficient group would not be different from day 35 value in control group).

Plasma phosphatidylcholine concentrations varied significantly between individuals (ranged between 1300 and $2000 \mu \mathrm{mol} /$ liter); however, when values were expressed as percent of the day 7 value (the end of a week on the standardized choline-containing diet) this intersubject variation was minimized. Plasma phosphatidylcholine (expressed as percent of day 7) decreased more than $10 \%$ on the average between days 1 and 7 (Fig. 2). In the control group, plasma phosphatidylcholine remained relatively constant between days 7 and 35 (91-100\% of day 7; Fig. 2). In the cholinedeficient group, plasma phosphatidylcholine decreased approximately $30 \%$ on the average during the 3-wk period when a choline-deficient diet was ingested (Fig. 2; $P<0.05$ that change in deficient group compared with control group was significant by two-sample $t$ test). When the cholinedeficient group was switched to a choline-sufficient diet during the last week of the study, plasma phosphatidylcholine returned to normal (Fig. 2; $P<0.01$ that day 35 value is different from day 28 value by $t$ test; day 35 value in deficient group is not different from day 35 value in control group). Erythrocyte membrane phosphatidylcholine rose $14 \%$ in control subjects and decreased $15 \%$ in deficient subjects between days 7 and $28(P<0.05$ that day 28 value is different from day 7 value by $t$ test).

Alanine aminotransferase is a liver-specific enzyme that is released into blood when hepatocytes are injured. The activity of this enzyme in serum remained relatively constant in the control group (0.3-0.4 $\mu \mathrm{mol} / \mathrm{liter}$ during the 5-wk study (Fig. 3). In the choline-deficient group, serum ALT activity increased steadily during the 3 -wk period when a choline-deficient diet was ingested (Fig. 3; Table 1; $P<0.05$ that change in the choline-deficient group as compared with the control group was significant by two sample $t$ test). All the choline-deficient subjects had an increase in serum ALT activity. When the deficient group was switched to a cholinesufficient diet during the last week of the study, serum ALT activity returned toward baseline (Fig. 3; $P<0.05$ that day 35 value is different from day 28 value by $t$ test; day 35 value in deficient group is not different from day 35 value in control group).

We observed an increase between days 7 and 28 in serum activities of several other enzyme markers for hepatocyte injury (AST, alkaline phosphatase) and in liver size; however, these changes did not achieve statistical significance. In both the control and deficient groups, there were no significant changes during the study in serum activities of other enzyme markers for hepatic damage (GGT, LDH) in tests measuring hepatic synthetic or conjugating activities (albumin, prothrombin time, partial thromboplastin time, total bilirubin, direct bilirubin), in tests of hepatic excretory capacity (bile acids), or in liver density (Table 1).

Serum total cholesterol decreased in all subjects (the semisynthetic diet contained no cholesterol). Total cholesterol in the serum of control subjects diminished to $3.57 \mathrm{mmol} /$ liter $( \pm 0.36)$ by day 14 and then rebounded to $4.27 \mathrm{mmol} /$ liter $( \pm 0.36)$ on day 28 . Total cholesterol in the serum of cholinedeficient subjects diminished to $3.90 \mathrm{mmol} / \mathrm{liter}( \pm 0.39)$ by day 14 , and then remained low $(3.96 \mathrm{mmol} / \mathrm{liter}[ \pm 0.31]$ on day 28). For this reason the change between days 7 and 28 in total serum cholesterol in the choline-deficient subjects was significantly greater than the change in total serum cholesterol in the control subjects $(P<0.01$ by two-sample $t$ test). These changes did not result from changes in serum HDL cholesterol, but rather from changes in LDL cholesterol (Table 1). Serum triglyceride concentrations did not change significantly in either group (Table 1). 


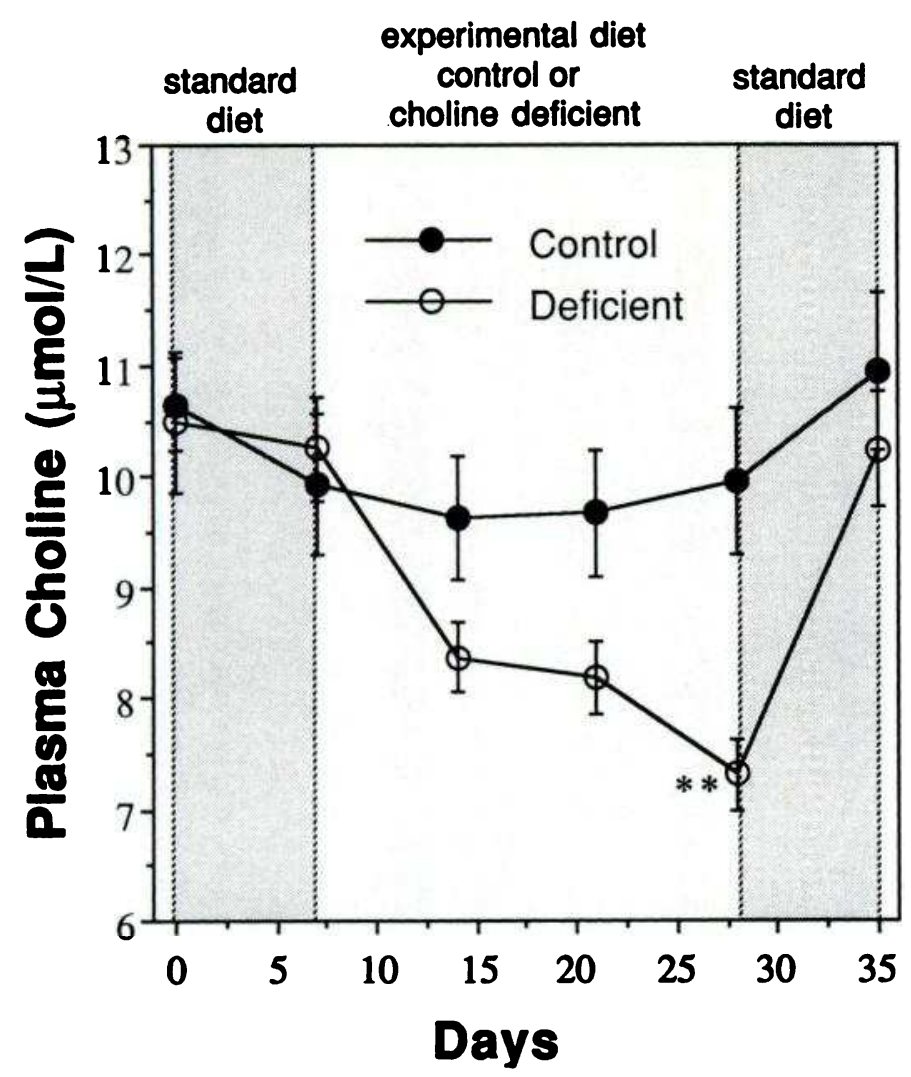

Figure 1. Plasma choline in humans ingesting a control or cholinedeficient diet. Healthy male subjects were hospitalized for 5 wk. During the first and last weeks of the study, all subjects consumed an identical choline-containing standard diet; during the middle 3 wk of the study subjects were divided into two groups: one that continued on the choline-containing diet, and one that consumed the same diet without choline. During the 5 th wk of the study all subjects consumed the choline-containing diet. Plasma choline was measured using a mass spectrometric method. Data are expressed as mean choline concentration $(\mu \mathrm{mol} / \mathrm{liter}) \pm \mathrm{SEM} .{ }^{* *}, P<0.01$ different from day 7 value.

Renal function, which was assessed using urinalysis, microscopic examination of urine, urine creatinine, serum creatinine, and blood urea nitrogen measurements did not change in either group during the study.

\section{DISCUSSION}

We designed the study to assess the effects of removing choline from the diet on three outcome measures. Plasma choline, plasma, and erythrocyte phosphatidylcholine were measured because they reflect the choline and choline ester content of tissues; serum ALT activity is the most commonly used clinical test for detecting liver injury. We observed that humans ingesting a choline-deficient diet for 3 wk had diminished plasma choline and phosphatidylcholine concentrations (Fig. 1 and Fig. 2) as well as diminished erythrocyte membrane phosphatidylcholine concentrations. Serum ALT activity increased significantly during the period when a choline-deficient diet was ingested (Fig. 3). The design of the study, as approved by our Institutional Review Board, was such that clinically significant changes in liver function (ALT activity exceeding $0.58 \mu \mathrm{kat} / \mathrm{liter}$ ) were not to be induced in the subjects. We suggest that choline deficiency of longer duration would have resulted in more prominent evidence of liver dysfunction (Fig. 3). Demands for choline are much higher in growing animals (1). The changes we observed in adult humans might have been greater in growing children.

Our control diet contained $500 \mathrm{mg}$ choline/day $(700 \mathrm{mg}$ of choline chloride). Total choline intake in the adult human is approximately $500-1000 \mathrm{mg} /$ day, with a large portion (approximately $400-800 \mathrm{mg}$ ) of this derived from phosphatidylcholine in foods. Consumption of choline will be higher in humans ingesting phosphatidylcholine (also called lecithin) as a dietary health food supplement.

The only source of choline other than diet is from the de novo biosynthesis of phosphatidylcholine catalyzed by phosphatidylethanolamine-N-methyltransferase (PeMT; EC 2.1.1.17). This enzyme synthesizes phosphatidylcholine via sequential methylation of phosphatidylethanolamine using S-adenosylmethionine as a methyl donor (16). Most PeMT activity is found in the liver (17), but significant activity is present in brain (18) and mammary gland (19) and detectable activity is found in most other tissues. There are no accurate estimates of the activity of phosphatidylethanolamine$\mathrm{N}$-methyltransferase in vivo. The best estimates, based on in vitro data, are that $15-40 \%$ of the phosphatidylcholine present in the liver is synthesized via the phosphatidylethanolamine- $\mathrm{N}$-methyltransferase pathway, with the remainder coming from the cytidine diphosphocholine pathway $(17,20)$. Choline deficiency is associated with increased hepatic PeMT activity measured in vitro $(21,22)$, but this is seen only when exogenous S-adenosylmethionine is added to the incubation mixture (23). The availability of $\mathrm{S}$-adenosylmethionine in the liver of choline-deficient animals limits the activity of this pathway $(24,25)$. Thus this pathway should not be able to compensate for diets deficient in choline unless the dietary content of methionine is high.

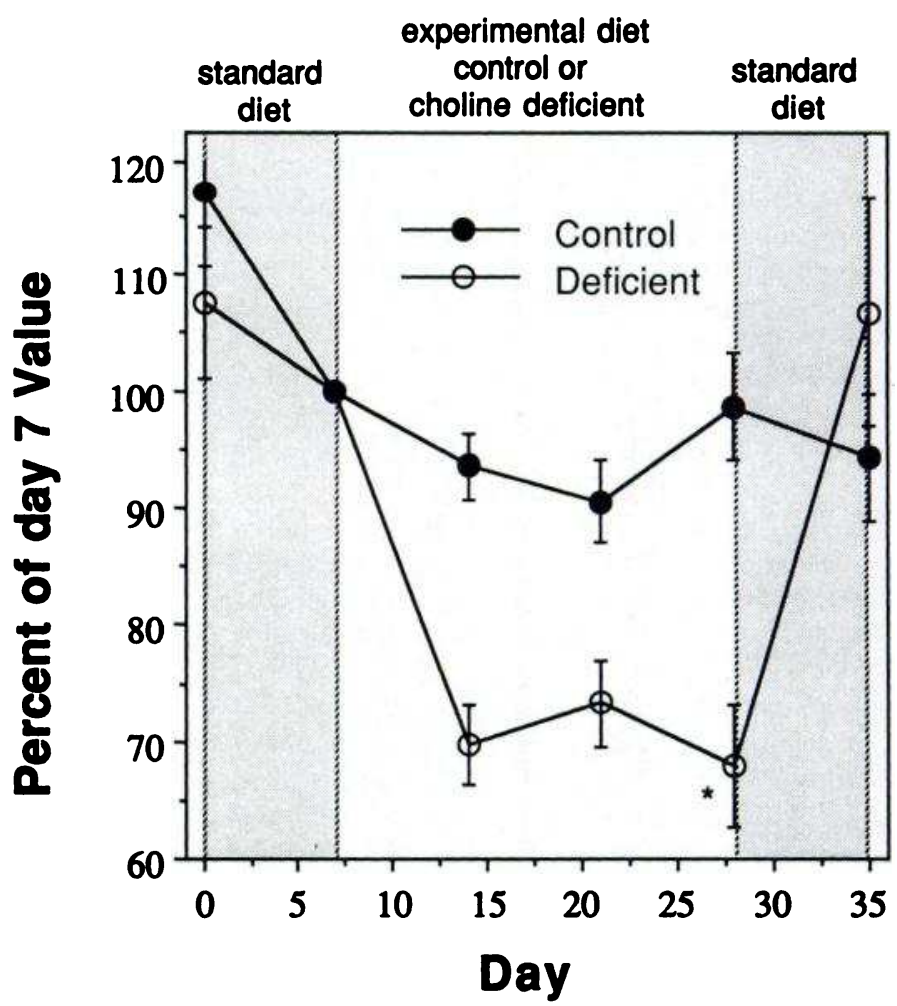

Figure 2. Plasma phosphatidylcholine in humans ingesting a control of choline-deficient diet. Subjects were treated as described for Fig. 1. Plasma phosphatidylcholine was measured using thin layer chromatography and phosphorus determination. Data are expressed as mean percent of day 7 phosphatidylcholine concentration \pm SEM. * $P<0.05$ different from day 7 value. 


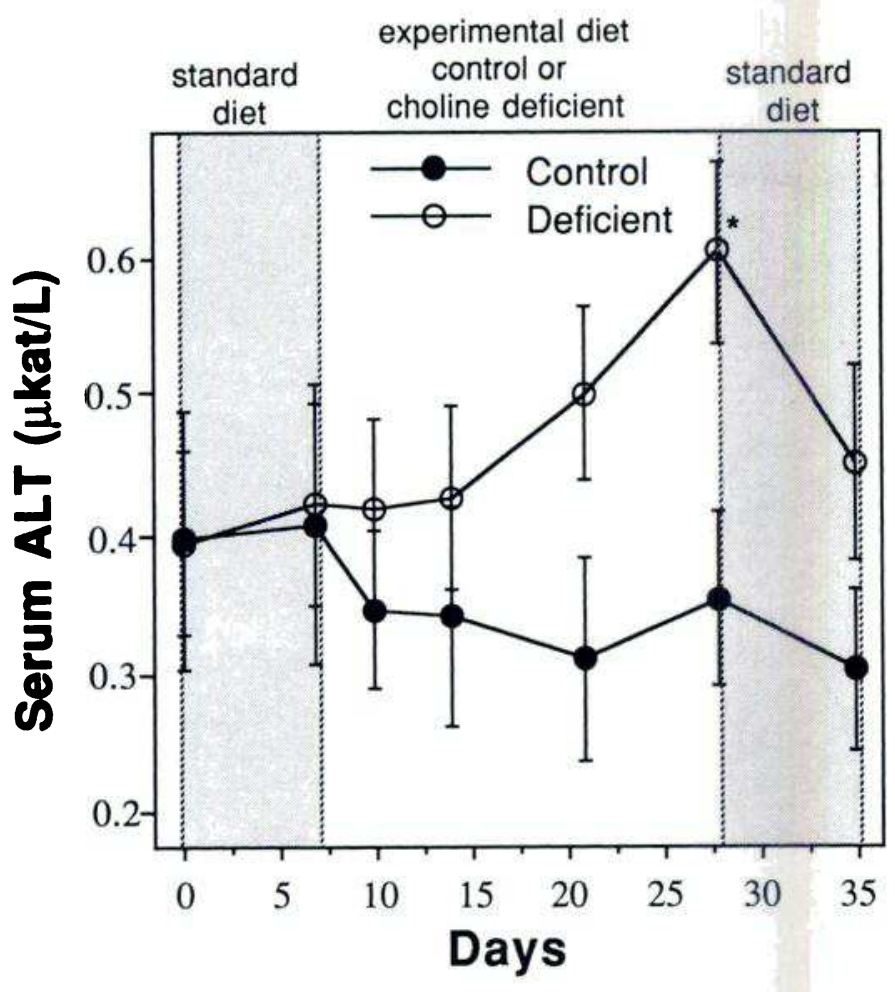

Figure 3. Serum alanine aminotransferase activity in humans ingesting a control or choline-deficient diet. Subjects were treated as described for Fig. 1. Serum alanine aminotransferase was determined using an automated spectrophotometric assay. Data are expressed as mean activity $(\mu$ kat/liter $) \pm$ SEM. ${ }^{*}, P<0.05$ different from day 7 value.

The demand for choline as a methyl donor is probably the major factor that determines how rapidly a diet deficient in choline will induce pathology. Our diet delivered the recommended daily allowance of methionine and folate. It is possible that if methionine were provided in larger amounts, the effects of removing choline from the diet would be moderated or eliminated. This is the case for adult rats (1). The pathways of choline and 1-carbon metabolism intersect at the formation of methionine from homocysteine (26). Methionine is regenerated from homocysteine in a reaction catalyzed by betaine:homocysteine methyltransferase, in which betaine, a metabolite of choline, serves as the methyl donor (26). Thus, when methionine availability is limited, the demand for choline may be increased.

In many mammals, chronic ingestion of a diet deficient in choline (and adequate though limited in methionine and folate content) has major consequences that include hepatic, renal, pancreatic, memory, and growth disorders. In the rat, hamster, guinea pig, pig, dog, monkey, trout, quail, and chicken, choline deficiency results in liver dysfunction (27). Hepatocyte turnover is greatly increased during choline deficiency secondary to hepatocyte injury $(28,29)$. We observed that serum ALT activity increased in choline-deficient subjects. These changes suggest that hepatocytes were injured in humans ingesting a choline-deficient diet. However, we did not observe any increase in serum activities of GGT and $\mathrm{LDH}$, two enzymes often released into blood when hepatocytes are damaged.

In animal models of choline deficiency large amounts of lipid (mainly triglycerides) accumulate in the liver, eventually filling the entire hepatocyte (30). Triacylglycerol ac- cumulation occurs because triglyceride must be packaged as very low density lipoprotein (VLDL) to be exported from the liver. Phosphatidylcholine is an essential component of VLDL (31, 32); other phospholipids cannot substitute (31, 32). Electron microscopic studies of hepatocytes from rats fed a choline-deficient diet have demonstrated ultrastructural abnormalities of the endoplasmic reticulum and Golgi system associated with delayed VLDL transport (33). Hepatocytes isolated from choline-deficient rats were unable to export VLDL until choline or methionine was made available (31). We did observe a significant decrease in serum cholesterol (derived from VLDL secreted by liver) in the choline-deficient subjects (Table 1). We did not observe a decrease in liver density or an increase in liver size, probably because such changes would occur only in moderate or severe fatty infiltration of the liver.

Choline deficiency may be of clinical importance in several groups of patients. The demand for choline in normal adults is likely to be smaller than the demand for choline in the infant, as large amounts of choline must be used to make phospholipids in growing organs (27). Malnourished humans, in whom stores of choline, methionine, and folate have been depleted $(6,7)$, are also likely to need more dietary choline than did our healthy adult subjects. We suggest that the effects of choline deficiency in infants or in malnourished patients would have been greater than those we observed in healthy adults who are not growing and have adequate body stores of choline. Bypass surgery involving large segments of the bowel (i.e., to produce weight loss in very obese humans) is associated with fatty liver. In obese rats that have had $90 \%$ of their small intestine bypassed, fatty liver develops. Choline supplementation prevents this, and choline-deficient diets in such animals exacerbate the accumulation of fat in the liver (34). Amino acid-glucose solutions used in total parenteral nutrition (TPN) of humans contain no choline (6, 35). The lipid emulsions used to deliver extra calories and essential fatty acids during total parenteral nutrition contain choline in the form of phosphatidylcholine (20\% emulsion contains $13.2 \mu \mathrm{mol} / \mathrm{ml}$ ), and we calculated that humans treated with TPN required 1000-1700 $\mu \mathrm{mol}$ (approximately 800-1360 mg) of choline-containing phospholipid per day during the first week of TPN therapy to maintain plasma choline levels (6). Burt et al. (9) reported that plasma choline concentrations were decreased in TPN patients at the same time that liver dysfunction was present. Conditions that enhance hepatic triglyceride synthesis (such as carbohydrate loading) increase the requirement for the choline for export of triglyceride from liver (36). Thus, treatment of malnourished patients with high-calorie TPN solutions when choline stores are depleted might enhance the likelihood of hepatic dysfunction. Some investigators have found that choline supplementation during TPN of rats prevents fatty liver (37), although others have not observed this effect (38). The definitive experiment, in which supplemental choline is administered and found to decrease the incidence of hepatic dysfunction during TPN in humans, has not yet been performed. Our study of normal humans suggests that such an experiment would be worthwhile.

In summary, we observed that when healthy humans consume a diet deficient in choline for 3 wk they deplete stores of choline in plasma and develop signs that suggest incipient liver dysfunction. It is likely that the demand for choline in humans will be influenced by the availability of methionine and folate in the diet. Our observations support the conclusion that choline is an essential nutrient for humans when excess methionine and folate are not available in the diet. 
We thank Ms Paula Gorman for her help as our research dietician. Some of the work described was supported by grants from the National Institutes of Health (HD-16727 and RR-00533) and by the U.S. Department of Agriculture (CRCR-1-2464). We would like to thank the Central Soya Company for providing us with soy protein.

\section{REFERENCES}

1. Zeisel, S. H. (1988) "Vitamin-like" molecules: choline. In Modern Nutrition in Health and Disease (Shils, M., and Young, V., eds) pp. 440-452, Lea \& Febiger, Philadelphia

2. Cohen, E. L., and Wurtman, R. J. (1975) Brain acetylcholine: increase after systemic choline administration. Life Sci. 16, 1095-1102

3. Haubrich, D. R., Wang, P. F., Clody, D. E., and Wedeking, P. W. (1975) Increase in rat brain acetylcholine induced by choline or deanol. Life Sci. 17, 975-980

4. Wecker, L. (1986) Neurochemical effects of choline supplementation. Can. J. Physiol. Pharmacol. 64, 329-333

5. Eagle, H. (1955) The minimum vitamin requirements of the $L$ and Hela cells in tissue culture, the production of specific vitamin deficiencies, and their cure. J. Exp. Med. 102, 595-600

6. Sheard, N. F., Tayek, J. A., Bistrain, B. R., Blackburn, G. L., and Zeisel, S. H. (1986) Plasma choline concentration in humans fed parenterally. Am. J. Clin. Nutr. 43, 219-224

7. Chawla, R. K., Wolf, D. C., Kutner, M. H., and Bonkovsky, H. L. (1989) Choline may be an essential nutrient in malnourished patients with cirrhosis. Gastroenterology 97, 1514-1520

8. Shapira, G., Chawla, R. K., Berry, C. J., Williams, P. J., Roy, R. G. B., and Rudman, D. (1986) Cysteine, tyrosine choline and carnitine supplementation of patients on total parenteral nutrition. Nutr. Int. 2, 334-339

9. Burt, M. E., Hanin, I., and Brennan, M. F. (1980) Choline deficiency associated with total parenteral nutrition. Lancet 2, 638-639

10. Tayek, J. A., Bistrian, B., Sheard, N. F., Zeisel, S. H., and Blackburn, G. L. (1990) Abnormal liver function in malnourished patients receiving total parenteral nutrition: a prospective randomized study. J. Am. Col. Nutr. 9, 76-83

11. Bremer, J., and Greenberg, D. (1961) Methyltransferring enzyme system of microsomes in the biosynthesis of lecithin. Biochim. Biophys. Acta 46, 205-216

12. Pomfret, E. A., daCosta, K. A., Schurman, L. L., and Zeisel, S. H. (1989) Measurement of choline and choline metabolite concentrations using high-pressure liquid chromatography and gas chromatography-mass spectrometry. Anal. Biochem. 180, 85-90

13. Zeisel, S. H., and daCosta, K. A. (1990) Choline determination using gas chromatography/mass spectrometry. J. Nutr. Biochem. 1, 55-59

14. Bligh, E. G., and Dyer, W. J. (1959) A rapid method of total lipid extraction and purification. Can. J. Biochem. Physiol. 37, 911-917

15. Kates, M. (1975) Techniques of lipidology. Laboratory techniques in biochemistry and molecular biology, $610 \mathrm{pp}$.

16. Blusztajn, J. K., Zeisel, S. H., and Wurtman, R. J. (1985) Developmental changes in the activity of phosphatidylethanolamine $\mathrm{N}$-methyltransferases in rat brain. Biochem. J. 232, 505-511

17. Bjornstad, P., and Bremer, J. (1966) In vivo studies on pathways for the biosynthesis of lecithin in the rat. J. Lipid Res. 7, 38-45

18. Blusztajn, J. K., Zeisel, S. H., and Wurtman, R. J. (1979) Synthesis of lecithin (phosphatidylcholine) from phosphatidylethanolamine in bovine brain. Brain Res. 179, 319-327
19. Yang, E. K., Blusztajn, J. K., Pomfret, E. A., and Zeisel, S. H. (1988) Rat and human mammary tissue can synthesize choline moiety via the methylation of phosphatidylethanolamine. Biochem. J. 256, 821-828

20. Sundler, R., and Akesson, B. (1975) Regulation of phospholipid biosynthesis in isolated rat hepatocytes. Effect of different substrates. J. Biol. Chem. 250, 3359-3367

21. Fallon, H. J., Gertman, P. M., and Kemp, E. L. (1969) The effects of ethanol ingestion and choline deficiency on hepatic lecithin biosynthesis in the rat. Biochim. Biophys. Acta 187, 94-104

22. Hoffman, D. R., Haning, J. A., and Cornatzer, W. E. (1981) Effects of a methyl-deficient diet on rat liver phosphatidylcholine biosynthesis. Can. J. Biochem. 59, 543-550

23. Schneider, W. J., and Vance, D. E. (1978) Effect of choline deficiency on the enzymes that synthesize phosphatidylcholine and phosphatidylethanolamine in rat liver. Eur. J. Biochem. 85, 181-187

24. Haines, D. S. (1966) The effects of choline deficiency and choline re-feeding upon the metabolism of plasma and liver lipids. Can. J. Biochem. 44, 45-57

25. Pascale, R., Pirisi, L., Daino, L., Zanetti, S., Satta, A., Bartoli, E., and Feo, F. (1982) Role of phosphatidylethanolamine methylation in the synthesis of phosphatidylcholine by hepatocytes isolated from choline-deficient rats. FEBS Lett. 145, 293-297

26. Finkelstein, J. D., Martin, J. J., Harris, B. J., and Kyle, W. E. (1982) Regulation of the betaine content of rat liver. Arch. Biochem. Biophys. 218, 169-173

27. Zeisel, S. H. (1990) Choline deficiency. J. Nutr. Biochem. 1, 332-349

28. Ghoshal, A. K., Ahluwalia, M., and Farber, E. (1983) The rapid induction of liver cell death in rats fed a choline-deficient methionine-low diet. Am. J. Pathol. 113, 309-314

29. Ghoshal, A. K., and Farber, E. (1984) The induction of liver cancer by dietary deficiency of choline and methionine without added carcinogens. Carcinogenesis 5, 1367-1370

30. Lombardi, B., Pani, P., and Schlunk, F. F. (1968) Cholinedeficiency fatty liver: impaired release of hepatic triglycerides. J. Lipid Res. 9, 437-446

31. Yao, Z. M., and Vance, D. E. (1988) The active synthesis of phosphatidylcholine is required for very low density lipoprotein secretion from rat hepatocytes. J. Biol. Chem. 263, 2998-3004

32. Yao, Z. M., and Vance, D. E. (1989) Head group specificity in the requirement of phosphatidylcholine biosynthesis for very low density lipoprotein secretion from cultured hepatocytes. $J$. Biol. Chem. 264, 11373-11380

33. Degertekin, H., Akdamar, K., Yates, R., Chen, I. I., Ertan, A., and Vaupel, R. (1986) Light and electron microscopic studies of diet-induced hepatic changes in mice. Acta Anat. 125, 174-179

34. Kaminski, D. L., Mueller, E. J., and Jellinek, M. (1980) Effect of small intestinal bypass on hepatic lipid accumulation in rats. Am. J. Physiol. 239, G358-G362

35. Chawla, R. K., Berry, C. J., Kutner, M. H., and Rudman, D. (1985) Plasma concentrations of transsulfuration pathway products during nasoenteral and intravenous hyperalimentaion of malnourished patients. Am. J. Clin. Nutr. 42, 577-584

36. Carroll, C., and Williams, L. (1982) Choline deficiency in rats as influenced by dietary energy somics. Nutr. Rep. Int. 25, 773

37. Kaminski, D. L., Adams, A., and Jellinek, M. (1980) The effect of hyperalimentation on hepatic lipid content and lipogenic enzyme activity in rats and man. Surgery 88, 93-100

38. Hall, R. I., Ross, L. H., Bozovic, M. G., and Grant, J. P. (1985) The effect of choline supplementation on hepatic steatosis in the parenterally fed rat. J. Parenter. Enterol. Nutr. 9, 597-599

Received for publication January 25, 1991. Accepted for publication February 5, 1991. 